\title{
WiFi TRANSMIT POWER AND ITS EFFECT ON CO-CHANNEL INTERFERENCE
}

\author{
Zyanya Cordova, Riddhi Rana, Giovanna Rendon, \\ Justin Thunell, and Abdelrahman Elleithy \\ Department of Computer Science, William Paterson University, \\ Wayne, New Jersey, USA
}

\begin{abstract}
The mass adoption of WiFi (IEEE 802.11) technology has increased numbers of devices simultaneously attempting to use high-bandwidth applications such as video streaming in a finite portion of the frequency spectrum. These increasing numbers can be seen in the deployment of highly-dense wireless environments in which performance can be affected due to the intensification of challenges such as co-channel interference (CCI). There are mechanisms in place to try to avoid sources of interference from non-WiFi devices. Still, CCI caused by legitimate WiFi traffic can be equally or even more disruptive, and also though some tools and protocols try to address CCI, these are no longer sufficient for this type of environment. Therefore, this paper investigates the effect of transmit power and direction have on CCI in a high-density environment consisting of multiple access points (APs) and multiple clients. We suggest improvements on publicly-existing documented power control algorithms and techniques by proposing a cooperative approach consisting of the incorporation of feedback from the receiver to the transmitter to allow it to reduce power level where possible, which will minimize the range of CCI for near clients without compromising coverage for the most distant ones.
\end{abstract}

\section{KEYWORDS}

Wi-Fi, co-channel interference, transmit power.

\section{INTRODUCTION}

The imposition of a short leash is no longer appealing in a world that is in constant motion, where the intangible is a vital part of everyday life. This dynamic is seen in the way we are connected and communicate with each other, which is shown in the constantly-growing use and dependency on wireless communication. Therefore, there are high demands for data traffic and data rates, which have led to the deployment of dense wireless networks and for which there is a wealth of information available on implementation strategies as well as the numerous challenges encountered [1] [2] [3]. IEEE 802.11 wireless devices operate in unlicensed bands of the Radio Frequency (RF) spectrum, and these bands, which are divided into channels, vary based on regional regulations. Because they are unlicensed bands, there can be significant interference from non-WiFi devices such as microwave ovens, Bluetooth devices, and radar systems, all of which also use these bands. A common technique is to avoid using channels that have excessive interference, which can be done by manual channel selection or specialized hardware that can both detect and identify the source and use an algorithm to choose a new channel with less interference [4].

Furthermore, WiFi technology also suffers from interference due to legitimate WiFi traffic from access points (APs) and end devices. This is because wireless is a shared media, and only one 
device can transmit on any given channel at any given time; otherwise, it will interfere with another device within the range that is attempting to do the same [3] [4]. Interference of this type is referred to as co-channel interference (CCI). CCI affects numerous wireless environments such as colleges, homes, corporate buildings, and stadiums due to the fact that these environments require high-density deployment that "causes overwhelming channel access contention at overloaded cells due to the carrier sense multiple access collision avoidance (CSMA/CA) protocol specified in the IEEE 802.11 standard for channel acquisition" [2]. This interference can be reduced by site surveys, channel selection, and manipulation of transmit power, the last solution being the focus of this paper [1] [3] [4] [5] [6].

The first step toward achieving a well-performing wireless LAN (WLAN), especially for highdensity environments, is performing pre and post-deployment site surveys and channel selection [1] [3] [4]. There are two types of surveys that can be performed when designing and deploying a wireless network. The initial site survey is performed before deployment, and it aids in identifying the best design to address coverage and demand. In contrast, the post-deployment survey uses statistics to validate and verify the already-deployed network [1] [2]. These site surveys are accomplished through the use of different tools such as Ekahau, Acrylic WiFi, and NetSpot products [7] [8] [9]. Large wireless designs require multiple APs that are arranged to ensure adequate coverage with no dead zones by creating slightly overlapping coverage areas [2]. In these surveys, the physical layout and even the furniture of the building in which the wireless network is going to be deployed are taken into consideration [2]. Using these tools, a plan can be developed by walking throughout the floor plan to identify walls, obstacles, glass divisions, and also recognize WiFi traffic, dead zones, and the best locations for APs[7] [8] [9]. Furthermore, if these APs are too close to each other and on the same channel, they will cause CCI. Similar to avoiding non-WiFi interference, wireless APs can have their channels selected manually or dynamically by using algorithms that avoid configuring adjacent APs to use the same channel. That solution is not always adequate since the regulating agencies are only able to provide a finite number of channels to use [10], as illustrated in [11, Figure 1]. For example, as seen in [12, Figure 2], for channels with a range of $22 \mathrm{MHz}$ in the $2.4 \mathrm{GHz}$ band, there is only sufficient space for channels one, six, and eleven, so they must be reused while avoiding interference. To avoid these interferences, protocols such as CSMA/CA and request to Send/Clear to Send (RTS/CTS) help avoid frame collision [13] [14]. There are also documented solutions, such as "Look Who's Talking (LWT), that allow for arbitrary schedules to be distributed to nodes in a WiFi network" [13, p.1], as well as most modern solutions such as beamforming [15], and multiple-input multiple-output technology [16], all of which are out of the scope of this paper.

In combination with proper channel selection, transmit power needs to be adjusted to minimize the chance of interfering with nearby APs on the same channel but without creating a coverage hole [17]. Increasing or decreasing transmit power will increase or reduce the range of each access point. The needed to transmit power will depend on many conditions, including the distance between APs, end-device antennas, and numerous other factors [17]. Instead of always transmitting at the maximum allowable power levels, which will lead to huge amounts of CCI, there is a power level that will provide the necessary coverage without unnecessarily interfering with nearby APs [17]. This can be accomplished with extensive measurements and manual tuning or with more effective methods that use algorithms and the signal levels from neighboring APs to dynamically select the level [17] [18] [19]. Dynamic approaches allow scalability and support for large environments with hundreds or thousands of APs, like college campuses and corporate office buildings that would not be practical with manual power level tuning [18]. 


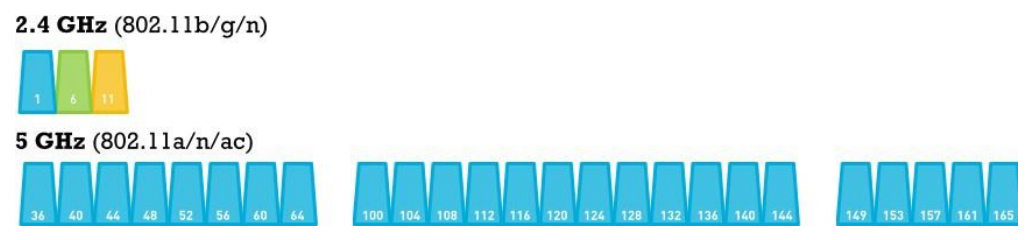

Figure 1. 2.4 Ghz and 5GHz non-overlapping channels

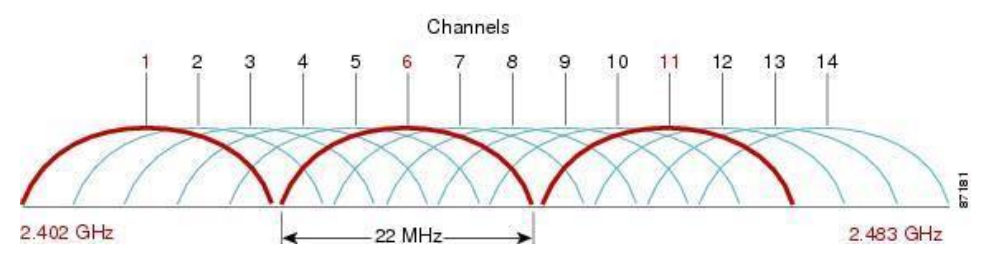

Figure 2. 2.4 Ghz channel plan

\section{Problem Identification}

All of these issues are compounded and require creative solutions to battle the CCI challenge due to the mass popularity of wireless, which sometimes entirely replaces wired infrastructure [1]. For example, many modern laptops do not even include a wired ethernet port. Furthermore, the high-volume and delay-intolerant use of streaming and real-time media like voice and video data stresses even well-designed wireless infrastructure [20]. Therefore, yet if all proper planning has been done, CCI can still arise from WiFi devices on other networks such as neighboring tenants and mobile hotspots, as well as the dependency on RTS/CTS algorithms without cooperation from separate networks [17]. Furthermore, power transmit is also key to tackling CCI, for example, in cases where two APs are on the same channel, and the area they must cover overlaps. They need to use their current power level to provide coverage at the outer edges [17].

This paper will focus on the single aspect of manipulating transmit power as a means to reduce CCI interference. Proprietary methods have been developed by wireless manufacturers, including Cisco's Transmit Power Control (TPC) algorithms and Aruba Adaptive Radio Management (ARM), to gather received signal strength of nearby APs and use this to determine proper power levels as illustrated in [18, Figure 3]. Other more recent solutions have been incorporated into the IEEE $802.11 \mathrm{n} / \mathrm{ac} / \mathrm{ax}$ standards that take seemingly more efficient approaches such as beamforming, as seen in [15, Figure 4] and the orthogonal frequency-division multiple access (OFDMA) resource units illustrated in [15, Figure 5] even though they require additional hardware and client feedback for the best results. By analyzing these solutions, this paper aims to improve on these existing solutions to the CCI challenge and incorporate external feedback into access point power control algorithms as a possible solution. 
International Journal of Computer Networks \& Communications (IJCNC) Vol.13, No.1, January 2021

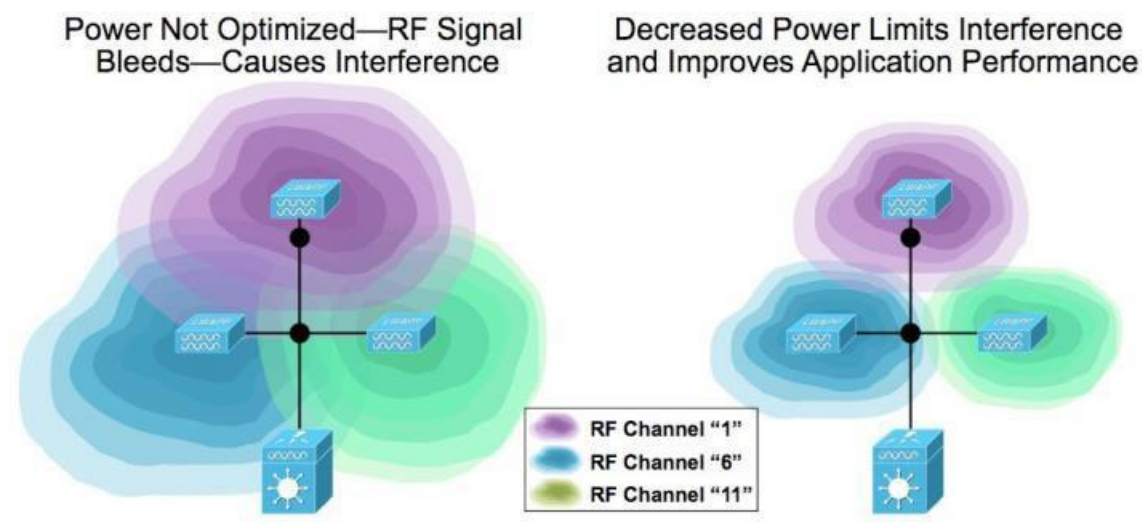

Figure 3. Transmit power control (TPC)

\section{RELATED WORK}

To manage the problem of WiFi co-channel interference, different solutions have been proposed regarding transmitting power control. Some of the solutions refer to new capabilities incorporated into the latest IEEE standard 802.11ax, such as Beamforming and BSS coloring. There are also proprietary solutions being offered, such as Cisco TPC and Aruba ARM, which are focused on centralized dynamic transmit power control, taking neighbor APs into consideration. Finally, there is a solution closely related to the proposed solution in this paper based on wireless link occupancy [16].

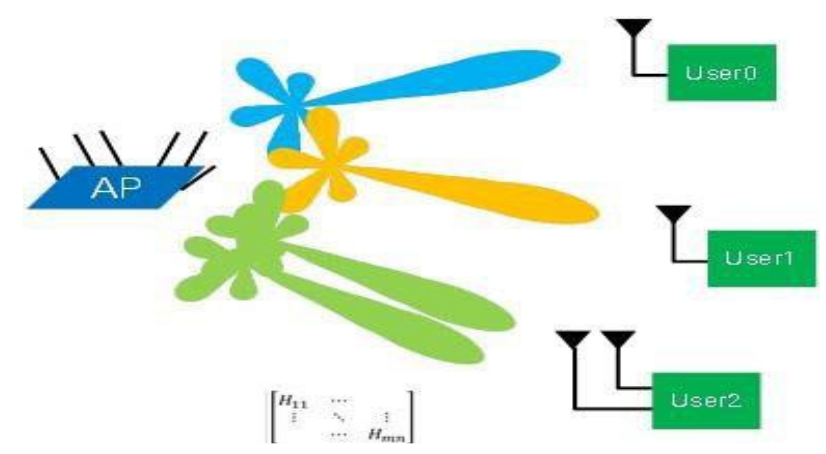

Figure 4. Beamforming technology

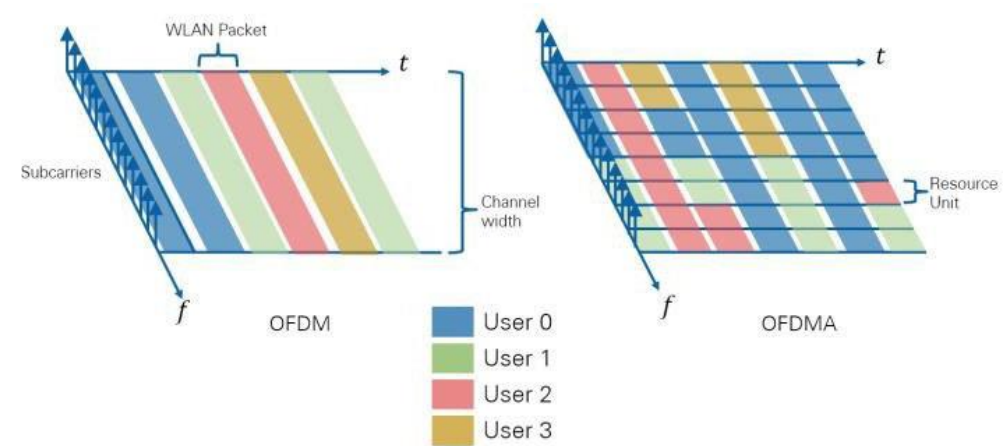

Figure 5. Multi-user orthogonal frequency-division multiple access 
Beamforming is a relatively recent innovation that can reduce CCI. Incorporated into the latest IEEE standard 802.11ax, this new capability uses multiple antennas along with receiver feedback to shape the direction of the transmission to be strongest in the direction of the receiver [1]. This allows APs to transmit to multiple devices at the same time as long as they are not aligned in the same direction from the source, as illustrated in [15, Figure 4]. With this solution, CCI is reduced because the signal is only sent where it is requested, which reduces contention in the areas that are not being transmitted to, and by the nature of transmitting to multiple clients at once, which reduces CCI by consuming less airtime [15]. However, this solution requires significant technical requirements to determine the location of each device and leads to overhead from communicating that location between each pair of sender/receiver.

Another thoroughly researched and new solution also based on 802.11ax is BSS coloring/spatial reuse. This solution alters standard clear channel assessment (CCA) rules by using various schemes to identify and ignore certain transmissions [22]. With BSS coloring, the CCA thresholds are altered for transmissions on other networks to allow for improved performance on overlapping networks, as illustrated in [23, Figure 6]. This allows for more simultaneous transmissions and therefore reduces CCI.

There are also proprietary solutions based on centralized dynamic transmit power control, such as Cisco TPC and Aruba ARM. Cisco TPC uses a centralized controller to dynamically set the power levels on all of the APs based on a list of received signals from each AP [18]. The calculations carried out to determine the transmit power are based on a user-defined threshold and the third loudest AP in the neighbor list. Cisco TPC offers two different methods to set the transmit power, which are TPCv1 and TPCv2. Cisco Networks published their TPC algorithms for TPCv1, which is known as coverage- optimized mode and is their recommended setting. Furthermore, TPCv1 is the mode that uses the third loudest neighbor, and it requires at least three APs to be within the range of each other to do the calculations; otherwise, the transmit power will stay at the maximum allowed power. In summary, the logic of these algorithms consists of choosing a lower level if three loud APs are detected since that would mean that there is already enough coverage; otherwise, a higher level is chosen; therefore, this set of algorithms "calculate the ideal power, evaluate a change recommendation, and implement a recommended power change" [18] as given in [24, Figure 7]. The dynamic calculations, along with the smoothing algorithm, slowly adjust all of the neighboring APs to find the right balance. Cisco also has a second mode, TPCv2, which is known as the interference-optimal mode and is recommended for areas of high interference. Its algorithm does not use the third neighbor, but it instead uses the neighbor list. Additionally, Aruba Networks, another wireless vendor, has similar but unpublished dynamic transmit power algorithms called ARM.

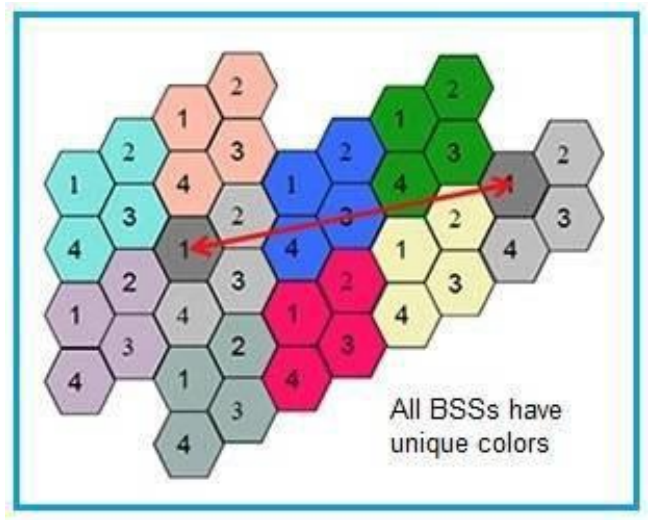

Figure 6. BSS coloring / spatial reuse 


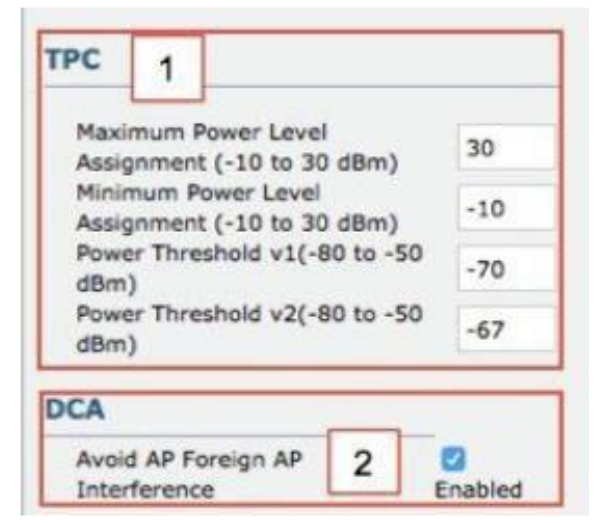

Figure 7. Cisco TPC

Finally, there is a paper called "Dynamic transmit-power control for WiFi access points based on wireless link occupancy" that proposes a solution that combines per-user power levels and data rate adjustments [16]. They also identify potential concerns related to a client's ability to detect/recommend $n$ discrete power levels, and accurate measurement of these signal levels is an essential part of their proposed tuning algorithm [16]. Professional research that leveraged actual, non-simulated hardware showed a significant opportunity for this approach.

\section{Proposed Solution}

After reviewing some of the existing solutions for co-channel interference focused on transmit power control, we determined that even enterprise-level products from the largest wireless manufacturers do not offer an all-encompassing solution to the dynamic transmit power problem; therefore, improvements are possible and needed in this area. Thereby, this paper proposes an enhancement where the AP is informed of a client's signal strength and uses this information to determine whether it can reduce power level. This will reduce the range of only those signals without affecting farther clients that need the full signal strength. This proposal would decrease the overall amount of time signals spend overlapped and would reduce CCI. Furthermore, this approach avoids the complex calculations found in beamforming and some of the issues raised in the "Dynamic transmit-power control for WiFi access points based on wireless link occupancy " paper.

The Client-driven Neighbor-friendly Opportunistic Power Reduction (CNOPR) solution proposed in this paper is based on the hypothesis that if there are slightly lower transmit levels that can be used for near clients without compromising their performance, then the signal will be weakened for distant clients sufficiently to become noise below CCA levels so that the far client will not have to wait to transmit, leading to reduced CCI. The model to be tested will calculate the radius for a given transmit power levels, and the difference in size for the radiuses where the signal is too weak to be considered for CCA is the potential CCI benefit. Figure 8 shows one scenario to which this solution would apply. Any environment that has overlapping networks on the same channel might benefit from this approach, and our model will attempt to validate this by determining the overlap of 'weak' signal areas. It is also important to note that this approach mostly benefits the 'other network,' but the benefit could be reciprocal if that network, as well, implements this solution.

To illustrate this solution and support the feasibility of the hypothesis, the overviews of transmit power and mathematical background that will be used for relevant calculations need to be presented. As noted previously, this solution to reduce CCI is based on transmit power, which not 
only determines the range of the signal but also plays a crucial role in lowering CCI as well as providing coverage and performance. Another technical concept that must be considered is the measurable energy lost by a radio wave as it creates distance between itself and its source [25]. Finally, we will also mention the signal-to-noise ratio, which shows what signals are usable by a receiver. Therefore, transmit power, free space path loss, and signal-to-noise ratio will be briefly introduced.

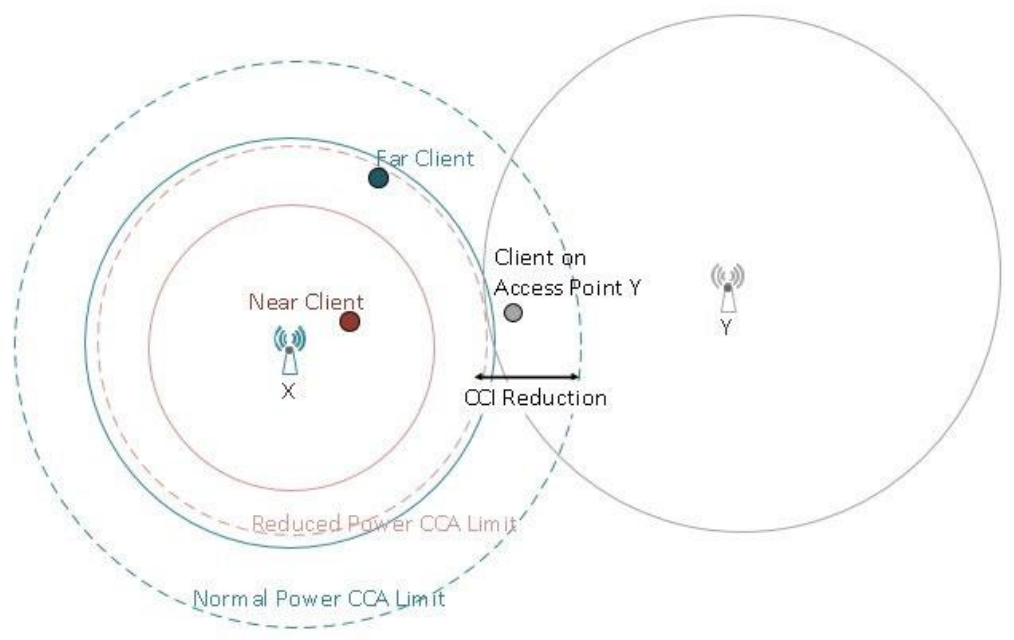

Figure 8. Sample model CNOPR

As might be expected, the stronger a signal is, the farther it will travel. The regulatory bodies that oversee the use of radio frequencies determine not only which frequencies can be used for whom and what, but also what are the maximum allowable energy levels. For WiFi, the maximum is often a value of 200 milliwatts [26], but even though this seems to be a small number, this signal level can be strong enough to overlap and interfere with nearby devices. Conversely, if a signal does not have enough strength, it will not cover the required area and will lead to coverage holes and reduced performance.

The next key concept involves the non-linear relationship between transmit power and distance,

which is determined by free-space path loss (FSPL). Free space path loss affects not only WiFi but all radio transmissions. It refers to the quantity of power lost by a signal traveling away from its transmitter [25]. There are two factors considered in the calculation of FSPL, which are radio frequency (f) and distance from the source (d). For this specific formula, a constant of free space, or open-air, is assumed. The value of the constant depends on the units of measurement used for (f) and (d). For example, when (d) is measured in kilometers and (f) in gigahertz, the value of the constant is 92.45 , and when (d) is in kilometers and (f) is in megahertz, the value is 32.45 , and there are other values as seen in [27, Table 1]. There are also variations for other media, such as traveling through water and different obstacles of varying densities such as walls and windows. In summary, the loss is not linear, and it is greater when there are obstacles present. It is also notable that higher frequencies suffer higher losses; therefore, a 2.4 gigahertz signal can travel farther away than a five gigahertz signal. As illustrated in [25, Figure 9], the signal loses 6 decibels whenever its distance from the source is doubled.

$\operatorname{FSPL}(\mathrm{dB})=20 \log (\mathrm{d})+20 \log (\mathrm{f})+\mathrm{K}(1)$ 
International Journal of Computer Networks \& Communications (IJCNC) Vol.13, No.1, January 2021

Values of constant $\mathrm{K}$ for different units of measurement for (f) and (d)

Table 1. Values of constant $\mathrm{K}$

\begin{tabular}{lll}
\hline Frequency (f) & Distance (d) & Constant (k) \\
\hline $\mathrm{MHz}$ & $\mathrm{Km}$ & 32.45 \\
\hline $\mathrm{GHz}$ & $\mathrm{Km}$ & 92.45 \\
\hline $\mathrm{KHz}$ & $\mathrm{m}$ & -87.55 \\
\hline $\mathrm{MHz}$ & $\mathrm{m}$ & -27.55 \\
\hline
\end{tabular}

The final technical background that needs to be discussed is the signal-to-noise ratio (SNR), which determines the usable signal. The signal-to-noise ratio takes into account the signal level and the noise floor [28]. The noise floor consists of very weak signals that the receiver cannot understand. It is important to note that the noise floor is not the same for each system, and within a particular system, it varies over time. However, the noise floor is usually around $-90 \mathrm{dBm}$. In general, an SNR of at least $20 \mathrm{~dB}$ is desired. Using typical values of $20 \mathrm{~dB}$ SNR and - $90 \mathrm{dBm}$ noise floor, the standard recommendation of $-70 \mathrm{dBm}$ is achieved, which is the minimum for basic services, as seen in [28, Figure 10]. Stronger signals are required for loss-sensitive services such as voice and video applications. Lower signals such as $-80 \mathrm{dBm}$ will hardly be able to offer any services, as shown in [28, Figure 11]. This concept plays an important role in the regulation of transmit power in order to reduce the impact of CCI because clear channel assessment rules (CCA rules) do not apply to low-level signals that fall under the noise floor. Thus, if the correct transmit power is applied, the intended receiver will obtain a usable signal, but this signal will not be strong enough for other distant, unrelated receivers to recognize it, thereby forcing them to wait before transmitting.

To model the proposed solution, a free Android application will be used to obtain sample signal levels. Some samples have already been gathered from the food court and David and Lorraine Cheng Library of William Paterson University, and the results showed that the CCI problem still exists beyond mere theory, as illustrated in [Figure 12]. As the next step, an application written in Java will be used to model signal strength versus distance using the FSPL equation and to calculate received signal level for near and far devices at different power levels. For the feasibility of the proposal, transmissions at a slightly reduced power for 'near' clients need to still be received strong, but when they reach 'far' clients, they should now be below CCA level.

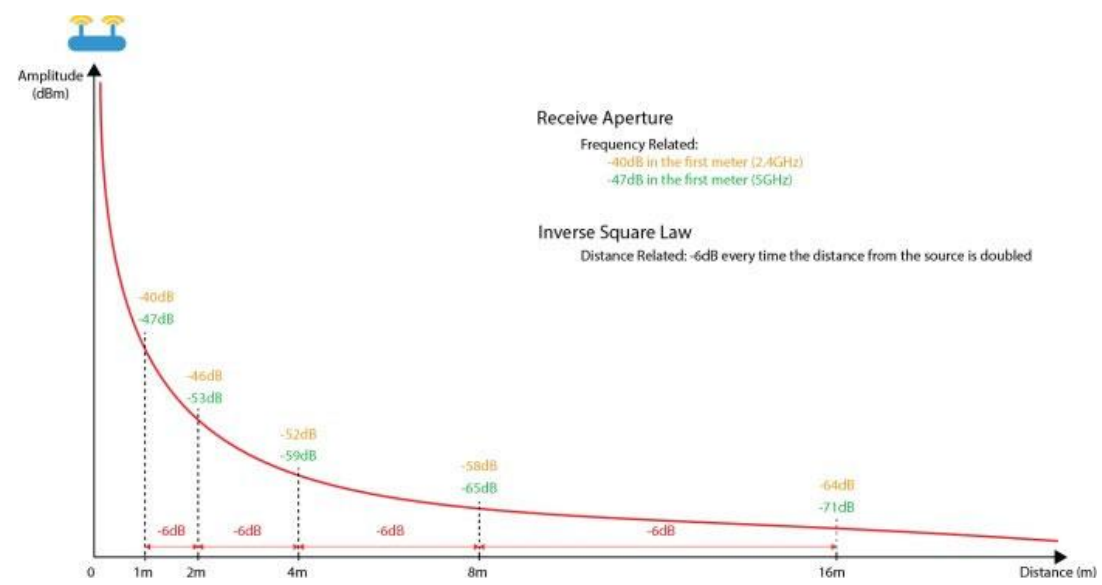

Figure 9. Free space path loss diagram 
International Journal of Computer Networks \& Communications (IJCNC) Vol.13, No.1, January 2021

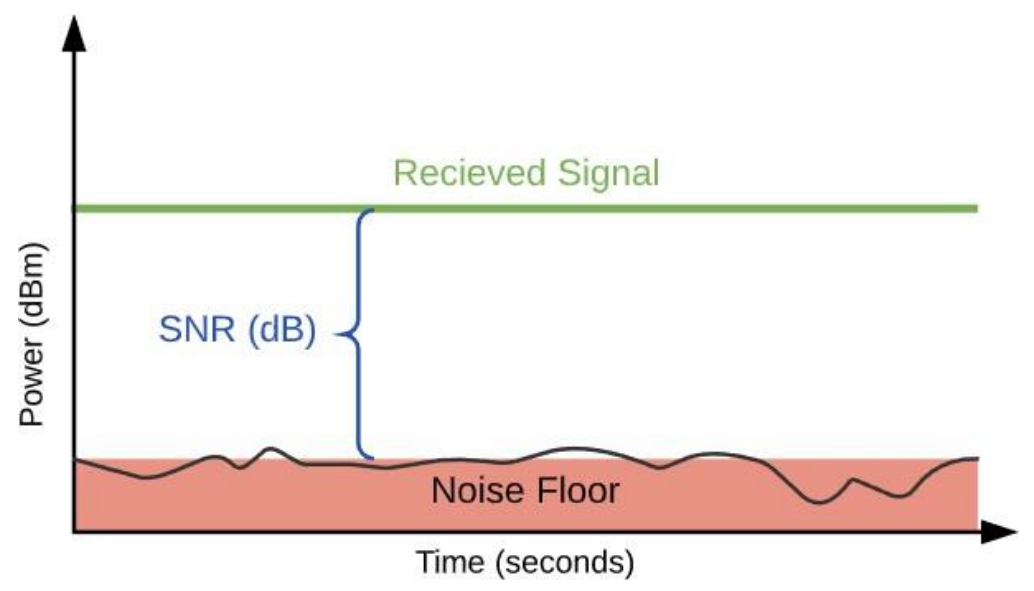

Figure 10. Signal-to noise ratio

Signal Strength

Required fo

$\begin{array}{ll}-70 \mathrm{dBm} & \begin{array}{l}\text { Minimum signal strength } \\ \text { for reliable packet delivery. Email, web }\end{array} \\ & \begin{array}{l}\text { Minimum signal strength } \\ \text { for basic connectivity. } \\ \text { Packet delivery may be } \\ \text { unreliable. }\end{array} \\ -80 \mathrm{dBm} \quad \mathrm{N} / \mathrm{A} \\ \begin{array}{l}\text { Approaching or drowning in } \\ \text { the noise floor. Any } \\ \text { functionality is highly } \\ \text { unlikely. }\end{array}\end{array}$

Figure 11. Signal-to noise ratio

As noted in the hypothesis of the CNOPR proposed solution of this paper, the reduction of CCI will be based on the reduction of transmit power levels in a way that the performance of a near client is not affected by this reduction and a distant client benefits from a signal below CCA level. In order to accomplish this, the method will reduce transmitted power levels until the point at which the signal is both CCA level for the distant client and strong for the near client. as seen in the algorithm scheme below. Figure 13 shows CNOPR implementation scheme. 
International Journal of Computer Networks \& Communications (IJCNC) Vol.13, No.1, January 2021

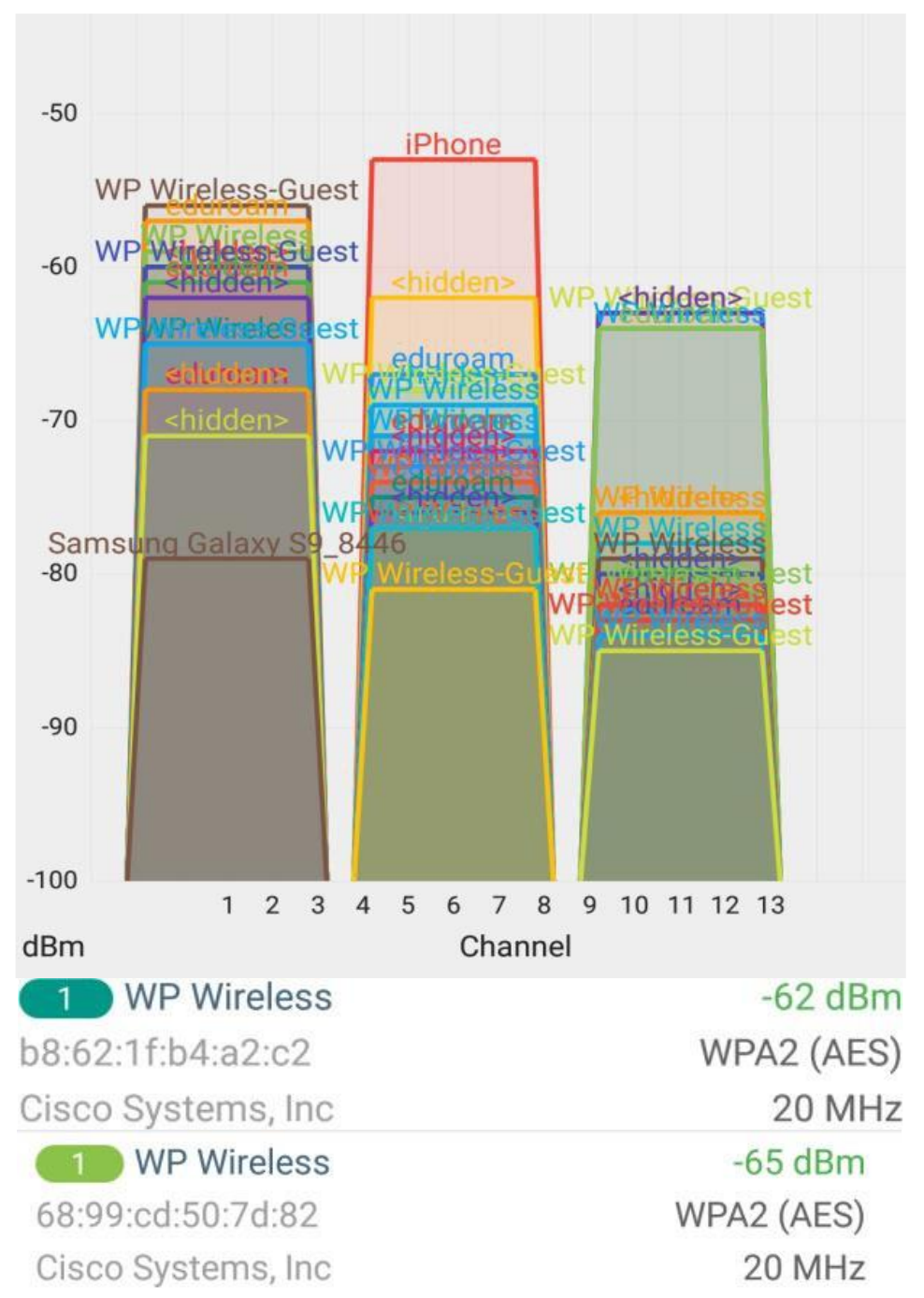

Figure 12. Sample signals from Cheng Library

Simplified implementation logic

\section{Client}

For every sample interval (' $x$ ' seconds)

If received power level from AP is above the predetermined threshold (a very strong signal)

Set 'Reduced Power Acceptable' flag in transmissions back to AP

If the power level is not strong enough and performance is/would be impacted

Unset 'Reduced Power Acceptable' flag in transmissions back to AP

AP

Use existing mechanisms for channel selection, and 'normal' transmit power

Send all transmissions at the normal level EXCEPT when a connected client has set the 'Reduced Power Acceptable' flag

While that flag is set, send transmissions to just that client at a slightly reduced power level. 
Note that the AP does not need to do any calculations and is dependent on a simple feedback flag from the client. The actual power reduction is predetermined. In our simulation, the reduction of 1 or 2 levels ( $3 \mathrm{dBm}$ each) produced the best results.

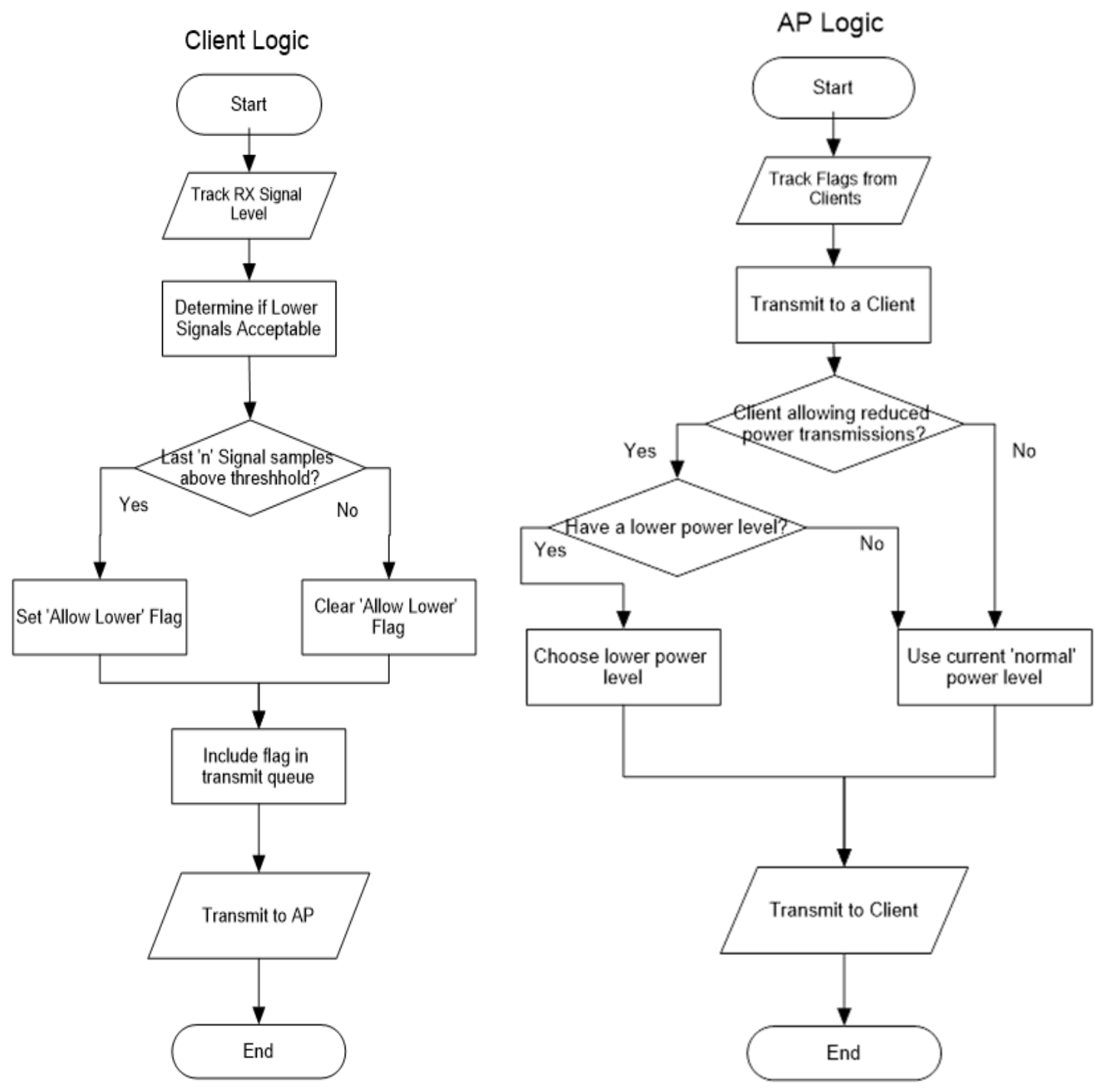

Figure 13. CNOPR implementation scheme

\section{Mathematical Model}

The mathematical model is based on the previously-noted hypothesis. Assuming that there are two clients between two APs, and one of them is near AP1 while the other is distant to one of the APs, there are slightly lower transmit power levels that can be used for clients within a strong signal level without compromising their performance; then the signal will be weakened for distant clients sufficiently to become noise below CCA level so that the distant client will not have to wait to transmit, leading to reduced CCI. Therefore, in order to obtain a mathematical model that supports this hypothesis, different variables were identified, and several assumptions were made regarding signal loss, channel, power levels, location, and clients. 
Assumptions:

1. Access points: two APs are considered in the mathematical model. The first one (AP1) has a near client (client1) connected to it, and the second AP (AP2) has a distant client (client2) connected to it.

2. Client1: there is a near client connected to AP1 in a near (strong) signal level range.

3. Client2: there is a second client considered in the model, which is a client located in a distant signal level range from AP1, and it is connected to AP2 at a minimum signal level range This is the client that should experience a CCI reduction obtained through this proposed solution.

4. Signal loss: the mathematical model for this proposed solution is supported by the FSPL formula, and as explained in point V, FSPL only accounts for signal loss through free space; therefore, calculations will not handle signal loss from other sources such as walls.

5. Channel: the two APs used for the calculations are on the same channel.

6. Power level: the two APs are using the same power level.

7. Location: to simplify the distance math in this model, it will be assumed that devices are all in a straight line.

8. Signal for client1: because the proposed solution hypothesizes that there is a lower transmit power for near clients that will reduce CCI, the near client is connected to an AP within a signal range generally accepted as strong $(-55 \mathrm{dBm})$.

9. Signal level for client2: it is assumed that the distant client is connected to a second AP within a signal range generally accepted as a minimum $(-75 \mathrm{dBm})$.

10. Power reduction: AP1 will reduce transmitted power for its near client (client1), which will potentially reduce CCI for its distant client (client2).

Inputs:

1. Frequency (f): in this model, both $2.4 \mathrm{GHz}$ and $5.0 \mathrm{Ghz}$ frequencies were considered.

2. Signal levels: four generally-acceptable signal levels were considered as seen in $[28$, table $1]$, ranging from a strong signal strength at $-55 \mathrm{dBm}$ to a CCA level at $-82 \mathrm{dBm}$. These signals were used to calculate distances and to determine when the model is effective.

3. Transmit power levels: eight different power levels were used for the calculation based on the Cisco 3700 series AP Transmit power levels [29], which have a $-3 \mathrm{dBm}$ difference between levels.

4. Signal level for a near client (client1): client1 is always at the strong signal level of AP1.

5. Signal level for the distant client (client2): client2 is always at the minimum signal level of AP2.

6. Distance between AP1 and client1: client1 is always at the same 'strong' signal level range of AP1; thus, client1's distance from AP1 remains fixed as well

Outputs or Dependent variables:

1. Distance between APs (apart): this model calculates the distance between the two APs, which is later increased in intervals to produce the results.

2. Distance between AP1 and client2 (d2): this distance is calculated for each interval/distance between APs. 
International Journal of Computer Networks \& Communications (IJCNC) Vol.13, No.1, January 2021

3. Signal levels received for both clients: these signals are calculated for each $n$ and $n+1$ power level for each interval of the distance between APs. These received signal levels determine when the model is effective.

Using the aforementioned assumptions and inputs, the solution was modeled in Java primarily using the FSPL formula to determine the received signals for both clients at various distances and power levels:

1. Power loss and distances were calculated with the following formulas:

$\operatorname{loss}=20 * \log ($ distance $)+20 * \log (\mathrm{f})-$ Constant distance $=10^{\wedge}((\operatorname{loss}-20 * \log ($ frequency $)$

- Constant ) / 20)

2. Original signals received, and post-power-reduction signals were calculated using the loss formula and the power levels $\mathrm{n}$ and $\mathrm{n}+1$ (next reduced level) as follows:

$\begin{array}{llll}\text { client1rxOrig } & =\text { powerN }- \text { loss (using distance client1 and freq) } \\ \text { client1rxLower } & =\text { powerLower }- \text { loss (using distance client1 and freq) } \\ \text { client2rxOrig } & =\text { powerN }- \text { loss (using distance client2 and freq) } \\ \text { client2rxLower } & =\text { powerLower }- \text { loss (using distance client2 and freq) }\end{array}$

3. AP overlap:

Overlap $=$ distance between AP1 and client2 / distance between AP1 and AP2

Overlap $=\mathrm{d} 2 /$ apart

\section{Simulation Results}

Based on thousands of iterations of the algorithm which determines the received power levels for both clients using FSPL formulas at various distances and power levels, we were able to classify the algorithm as effective or ineffective along with a reason and summary. The overlap statistic used in the results (distance between AP1 and client2 / distance between AP1 and AP2 ) removes the actual distance from the results and focuses on AP overlap; however, as expected, $2.4 \mathrm{GHz}$ distances were greater than the corresponding $5 \mathrm{GHz}$ distances. The detailed simulation results are given in Table 2. 
International Journal of Computer Networks \& Communications (IJCNC) Vol.13, No.1, January 2021

\begin{tabular}{|c|c|c|c|}
\hline Results & Sample1 & Sample2 & Sample3 \\
\hline $\begin{array}{l}\text { Freq } \\
(\mathrm{GHz})\end{array}$ & $2.4 \mathrm{GHz}$ & $2.4 \mathrm{GHz}$ & $2.4 \mathrm{GHz}$ \\
\hline $\begin{array}{l}\text { TxLevels } \\
\text { (Index) }\end{array}$ & $0>6$ & $1 \quad->3$ & $1>4$ \\
\hline $\begin{array}{l}\text { Tx->TxReduced } \\
(\mathrm{dBm})\end{array}$ & $23>5$ & $20->14$ & $20>11$ \\
\hline $\begin{array}{l}\text { Apart } \\
(\mathrm{m})\end{array}$ & 2370 & 2160 & 1005 \\
\hline $\begin{array}{l}\mathrm{d} 1 \\
(\mathrm{~m})\end{array}$ & 78.9 & 55.9 & 55.9 \\
\hline $\begin{array}{l}\mathrm{rx} 1 \\
(\mathrm{dBm})\end{array}$ & -55 & -55 & -55 \\
\hline $\begin{array}{l}\text { rxiLower } \\
(\mathrm{dBm})\end{array}$ & -73 & -61 & -64 \\
\hline $\begin{array}{l}\mathrm{d} 2 \\
(\mathrm{~m})\end{array}$ & 1580.6 & 1601.2 & 446.2 \\
\hline $\begin{array}{l}\mathrm{rx} 2 \\
(\mathrm{dBm})\end{array}$ & -81 & -84.1 & -73 \\
\hline Overlap & $66.70 \%$ & $74.10 \%$ & $44.40 \%$ \\
\hline Effectiveness & Ineffective & Not applicable & Effective \\
\hline
\end{tabular}

Table 2. Snippet of detailed simulation results

The aggregated data shown in Table 3. It can be seen that the model tracks the number of samples and the minimum and maximum overlap observed for each result using a key of \{frequency, power level drop, and result $\}$ - this reduced tens of thousands of results into a very small number of groups.

\begin{tabular}{|c|c|c|c|}
\hline Category & $\begin{array}{l}\text { Sample } \\
\text { Size }\end{array}$ & $\begin{array}{l}\text { Min } \\
\text { Overlap }\end{array}$ & $\begin{array}{l}\text { Max } \\
\text { Overlap }\end{array}$ \\
\hline $\begin{array}{l}2.4 \mathrm{GHz} \text { reduced } 1 \text { power } \\
\text { levels, Effective }\end{array}$ & 322 & $61.3 \%$ & $69.1 \%$ \\
\hline $\begin{array}{l}2.4 \mathrm{GHz} \text { reduced } 1 \text { power } \\
\text { levels, Not applicable }\end{array}$ & 609 & $69.1 \%$ & $77.6 \%$ \\
\hline $\begin{array}{l}2.4 \mathrm{GHz} \text { reduced } 2 \text { power } \\
\text { levels, Ineffective: } \\
\text { client } 2 \text { still hears }\end{array}$ & 1000 & $0.1 \%$ & $52.9 \%$ \\
\hline $\begin{array}{l}2.4 \mathrm{GHz} \text { reduced } 2 \text { power } \\
\text { levels, Not applicable }\end{array}$ & 585 & $69.1 \%$ & $77.6 \%$ \\
\hline $\begin{array}{l}5.0 \mathrm{GHz} \text { reduced } 1 \text { power } \\
\text { levels, Effective }\end{array}$ & 155 & $61.3 \%$ & $69.1 \%$ \\
\hline $\begin{array}{l}5.0 \mathrm{GHz} \text { reduced } 1 \text { power } \\
\text { levels, Not applicable }\end{array}$ & 294 & $69.2 \%$ & $77.7 \%$ \\
\hline $\begin{array}{l}\text { 5.0GHz reduced } 4 \text { power } \\
\text { levels, Ineffective: weak } \\
\text { signal for client } 1\end{array}$ & 869 & $0.3 \%$ & $77.7 \%$ \\
\hline
\end{tabular}

Table 3: Aggregated data from simulation results 
Based on the above summaries:

1. The model is ineffective when the APs are very close together. This is because the signal cannot be reduced enough so that the other device will not continue to hear it above the CCA level, and it is summarized as ineffective because it is still heard by client2. These samples had an 'overlap range' starting at zero and going up to $44 \%$ or $\sim 60 \%$ based on the amount of power reduction.

2. The model is also ineffective or not applicable when the APs are very far apart because they already were not causing CCI, and it is summarized as not applicable because it is already below CCA. These samples had 'overlap ranges' above $69 \%$.

3. The last category for ineffective is when the signal is reduced too much to the point that it is now too weak for the near client, and even though it falls below CCA for the distant client, the signal is so weak that it compromises performance for the near client.

4. The effective category, summarized as effective, represents a slight overlap, and it has an 'overlap range' of $44 \%$ to $\sim 70 \%$, which implies that the distant client heard the signal just loudly enough to factor into CCA calculations, and the small reduction means it no longer has to. Therefore, it reduces CCI.

5. The summarized data also showed that the highest overlap in the results was $77 \%$. However, this is due to the assumptions we made for client2's positioning, which are that client2 is always between AP1 and AP2, and it is positioned at the edge of AP2's minimum power level range. If client 2 were on the far side of AP2, the overlap would be greater than $100 \%$, and as seen in the results, those would all be already below CCA.

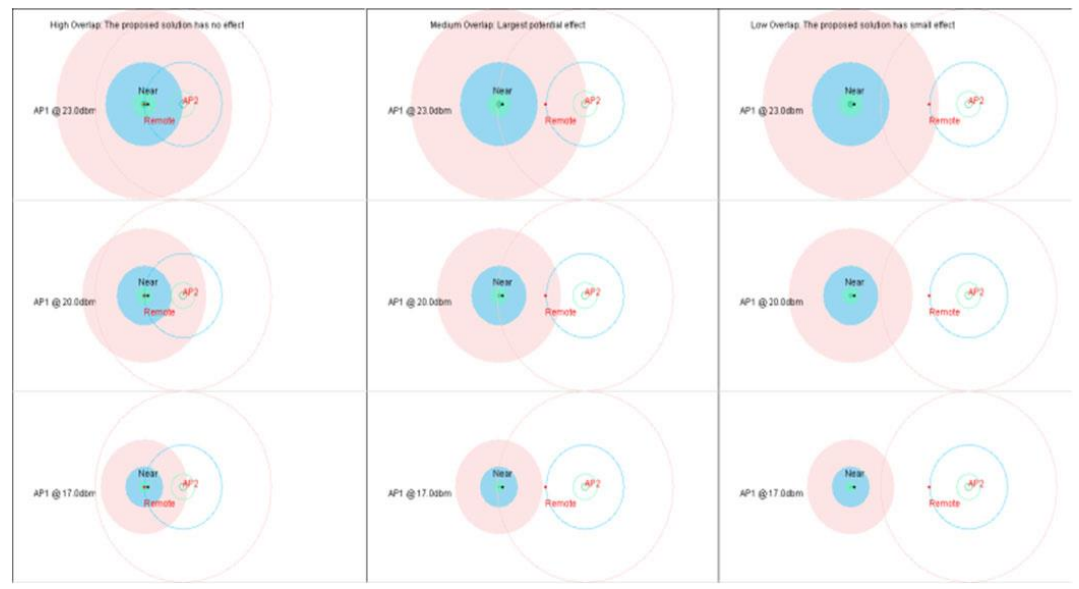

Figure 14. Diagram for sample position and ranges

\section{Conclusion}

The simulation results confirmed that there are multiple combinations of AP and client locations that will result in lowered CCI for clients on remote APs that are in the overlapping signal area. In our model, we used relatively fixed positions in which the near client is always within AP1's strong signal level at the initial power level, and the remote AP's client is positioned between the two APs in a straight line. As seen in the results, the patterns clearly show that the most effective area for this model to produce a reduction of CCI is when there is a medium overlap between two APs. If the APs are too close, then the distant client will always hear the signal regardless of the reduction. If the APs are too far apart, there is already no CCI. It also shows that sometimes even greater gains can be obtained by reducing power by more than one power level; however, finding the balance and a perfect number would be considered future work. 
International Journal of Computer Networks \& Communications (IJCNC) Vol.13, No.1, January 2021

A more complex model could more accurately predict the potential benefits by doing real math for intersecting circles. Further modeling work could also involve simulating traffic and doing a pre-and post-implementation baseline or allowing for more accurate loss calculations than pure FPSL, as seen in various predictive site survey tools that account for loss due to walls and other sources of attenuation. Another consideration would be measuring real-world layouts and seeing how many systems would likely be in the overlap areas and would benefit from this solution. The most extensive testing option would skip modeling and implement the logic in real systems and use real traffic flows.

\section{CONFLICTS OF INTEREST}

The authors declare no conflict of interest.

\section{ACKNOWLEDGMENTS}

The authors acknowledge the Department of Computer Science in William Paterson University for using departmental resources in for conducting this research.

\section{REFERENCES}

[1] M. Guessous and L Zankouar, "Cognitive directional cost-based transmit power control in IEEE 802.11 WLAN," in 2017 Int. Conf. Information Networking (ICOIN), Da Nang, Vietnam, 11-13 Jan. 2017. [Online]. Available: https://ieeexplore.ieee.org/document/7899520

[2] Phillip B. Oni and Steven D. Blostein, "Decentralized AP selection in large-scale wireless LANs considering multi-AP interference," in 2017 Int. Conf Computing, Networking and Communications (ICNC), Santa Clara, California, United States, 26-29 Jan. 2017. [Online]. Available: https://arxiv.org/pdf/1606.02316.pdf

[3] A. M. Voicu, L. Lava, L. Simić, and M. Petrova, "The Importance of adjacent channel interference: experimental validation of ns-3 for dense WiFi networks," in MSWiM '17 - The 20th ACM Int. Conf. Modeling, Analysis and Simulation of Wireless and Mobile Systems, 21 Nov. 2017, Miami Beach, Florida, United States. [Online]. Available: https://dl.acm.org/citation.cfm?id=3127548

[4] M. H. Dwijaksara, W. S. Jeon, and D. G. Jeong, "A centralized channelization scheme for wireless LANs exploiting channel bonding," in SAC 2018 - The 33rd Ann. ACM Symp. Applied Computing, 9 April 2018. [Online]. Available: https://dl.acm.org/citation.cfm?id=3167357.

[5] A. King and U. Roedig, "Differentiating clear channel assessment using transmit power variation," ACM Transactions on Sensor Networks (TOSN), vol. 14, no. 2, July 2018. [Online]. Available https://dl.acm.org/doi/10.1145/3209044

[6] Y. Zhang, S. Li, Z. Shang, and Q. Zhang, "Performance analysis of IEEE 802.11 DCF under different channel conditions," in 2019 IEEE 8th Joint Chongqing, China, 24-26 May 2019. [Online]. Available: https://ieeexplore.ieee.org/stamp/stamp.jsp?arnumber=8785766.

[7] "Ekahau Connect ${ }^{\mathrm{TM}}$,"Ekahau. [Online]. Available: https://www.ekahau.com/products/ekahauconnect/overview/.

[8] "WiFi planning and WiFi site survey with Acrylic Wi-Fi Heatmaps," Acrylic WiFi. [Online]. Available: https://www.acrylicwifi.com/en/wlan-wifi-wireless-network-software-tools/wifi-sitesurvey-software-acrylic-heat-maps

[9] "FREE WiFi Site Survey Software for MAC OS X \& Windows," NetSpot, 03-Oct-2019. [Online]. Available: https://www.netspotapp.com/

[10] P. Li, N. Scalabrino, Y. Fang, E. Gregori, and I. Chlamtac, "Channel interference in IEEE 802.11b systems," in IEEE GLOBECOM 2007 - IEEE Global Telecommunications Conf. Washington, DC, United States, 26-30 Nov. $2007 . \quad$ [Online]. Available: http://www.fang.ece.ufl.edu/mypaper/globecom07li.pdf.

[11] "Designing a Dual-Band Wireless Network," MetaGeek. [Online]. Available: https://www.metageek.com/training/resources/design-dual-band-wifi.html 
International Journal of Computer Networks \& Communications (IJCNC) Vol.13, No.1, January 2021

[12] "Channel Planning Best Practices," Cisco Meraki. [Online]. Available: https://documentation.meraki.com/MR/WiFi_Basics_and_Best_Practices/Channel_Planning_Best_Pr a ctices

[13] C. F. Shih, Y. Jian, and R. Sivakumar, "Look who's talking: a practical approach for achieving scheduled WiFi in a single collision domain," in CoNEXT 2015 - Int. Conf. on emerging Networking EXperiments and Technologies, 1 Dec. 2015, Heidelberg, Germany. [Online]. Available: https://dl.acm.org/citation.cfm?id=2836116

[14] J. Zhang, G. Han, and Y. Qian, "Queuing theory based co-channel interference analysis approach for high-density wireless local area networks," Sensors, vol. 16, 23 Aug. 2016. [Online]. Available doi: 10.3390/s16091348, https://www.ncbi.nlm.nih.gov/pmc/articles/PMC5038626/

[15] "Introduction to 802.11ax High-Efficiency Wireless," National Instruments. [Online]. Available: http://www.ni.com/en-us/innovations/white-papers/16/introduction-to-802-11ax-high-efficiencywireless.html

[16] C. Gandarillas, C. M. Engeños, H. L. Pombo, and A. G. Marques, "Dynamic transmit-power control for WiFi access points based on wireless link occupancy," in 2014 IEEE Wireless Communications and Networking Conference (WCNC), 6-9 April 2014. Istanbul, Turkey. [Online]. Available: https://ieeexplore.ieee.org/document/6952281

[17] C. H. Hsu, C. T. Yu, and T. Y. Wang, "Transmit power allocation for distributed spectrum sensing in the presence of co-channel interference," in 2018 3rd Int. Conf. Computer and Communication Systems (ICCCS), 27-30 April 2018, Nagoya, Japan. [Online]. Available: https://ieeexplore.ieee.org/document/8463332

[18] "Radio Resource Management White Paper - Transmit Power Control (TPC) Algorithm [Cisco 5500 Series Wireless Controllers]," Cisco, 24- Jun-2016.[Online].Available: https://www.cisco.com/c/en/us/td/docs/wireless/controller/technotes/8-

3/b_RRM_White_Paper/b_RRM_White_Paper_chapter_0101.html

[19] M. Kasslin and A. Lappeteläinen , "Transmitter power control (TPC) for 802.11 WLAN - Rev.1," Nokia Research Center, July 2000. [Online]. Available: http://www.ieee802.org/11/Documents/DocumentArchives/2000_docs/01908E-TPC-for-802.11-pptRev1.ppt

[20] D. M. Souza, F.B. D. L. Neto, and E. D. Q. Albuquerque, "Projecting IEEE 802.11n WLAN networks with cultural algorithms for VoIP implementation," in 2018 IEEE Latin American Conf. Computational Intelligence (LA-CCI), 7-9 Nov. 2018, Guadalajara, Mexico. [Online]. Available: https://ieeexplore.ieee.org/document/8625253

[21] "Adaptive Radio Management (ARM)," Aruba Networks. [Online]. Available: https://www.arubanetworks.com/techdocs/ArubaOS_60/UserGuide/ARM.php

[22] Korov, Evgeny et all. "A tutorial on IEEE 802.11ax high efficiency WLANs." IEEE Communications Surveys and Tutorials, Vol. 21, No. 1, First Quarter2019. [Online]. Available: https://ieeexplore.ieee.org/stamp/stamp.jsp?arnumber=8468986

[23] Jun, Xian Ke. "[New WiFi] 802.11ax BSS coloring." Castle on a Cloud. 23 Dec. 2018. [Online]. Available: https://note-on-clouds.blogspot.com/2018/12/wifi-80211ax-bss-coloring.html

[24] "Enterprise mobility 8.5 design guide." Cisco. [Online]. Available: https://www.cisco.com/c/en/us/td/docs/wireless/controller/8-5/Enterprise-Mobility-8-5-DesignGuide/Enterprise_Mobility_8-5_Deployment_Guide/wlanrf.html

[25] Vergès, François. "Free space path loss diagrams." SemFio Networks. April 1. 2018. [Online]. Available: https://www.semfionetworks.com/blog/free-space-path-loss-diagrams

[26] Nefkens, Patrick. "Maximum allowed transmission power in the ETSI domain." Dutch-Fi. 7 Aug. 2017. [Online]. Available: https://dutch-fi.eu/2017/08/maximum-allowed-transmission-power-in-theetsi-domain/

[27] Poole, Ian. "Free space path loss: details \& calculator." Electronics Notes. [Online]. Available: https://www.electronics-notes.com/articles/antennas-propagation/propagation-overview/free-spacepath-loss.php

[28] "Signal-to-noise ratio (SNR) and wireless signal strength." Cisco Meraki. [Online]. Available: https://documentation.meraki.com/MR/WiFi_Basics_and_B est_Practices/Signal-toNoise_Ratio_(SNR)_and_Wireles_Signal_Strength

[29] "Cisco Aironet 3700 Series Access Points Data Sheet." Cisco. Updated March 22, 2019. [Online]. Available: $\quad$ https://www.cisco.com/c/en/us/products/collateral/wireless/3700-series-accesspoint/data_sheet_c78-729421.html 